\title{
Treatment of bronchomalacia using three-dimensional printed polycaprolactone scaffold in a pediatric patient
}

Lei Wang, PhD, ${ }^{\mathrm{a}}$ Wenhao Liu, MD, ${ }^{\mathrm{a}}$ Jiankang He, PhD, ${ }^{\mathrm{b}}$ Xiaofei Li, MD, ${ }^{\mathrm{a}}$ and Lijun Huang, MD, ${ }^{\mathrm{a}} \mathrm{Xi}$ ' an, China

\footnotetext{
From the a Department of Thoracic Surgery, Tangdu Hospital, Fourth Military Medical University, Xi'an, China and 'State Key Laboratory for Manufacturing Systems Engineering, Xi'an Jiaotong University, Xi'an, China. This work was supported by the Clinical Research Innovation Major Fund (2015LCYJ003).

Disclosures: Authors have nothing to disclose with regard to commercial support.

Received for publication Oct 12, 2018; revisions received Nov 7, 2018; accepted for publication Nov 17, 2018; available ahead of print Jan 29, 2019.

Address for reprints: Lijun Huang and Xiaofei Li, Department of Thoracic Surgery, Tangdu Hospital, Fourth Military Medical University, 577\#, Xinsi Rd, Xi'an, China) (E-mail: 1xfchest@fmmu.edu.cn or tuodi1986@126. com).

J Thorac Cardiovasc Surg 2019;157:e287-90

$0022-5223 / \$ 36.00$

Copyright (C) 2018 by The American Association for Thoracic Surgery

https://doi.org/10.1016/j.jtcvs.2018.11.095
}

Video clip is available online.

Bronchomalacia, a weakness of the bronchus due to the reduction and/or atrophy of the longitudinal elastic fibers or impaired cartilage integrity, is a rare respiratory disease in children. Green and colleagues ${ }^{1,2}$ first used a 3-dimensional printed (3DP) polycaprolactone (PCL) scaffold to suspend the malacic bronchus and achieved excellent therapeutic results. However, few cases have been reported on the application of this technology or the clinical features of scaffold in vivo. Herein, a pediatric patient with left mainstem bronchomalacia was cured using the 3DP PCL scaffold technique.

\section{CASE REPORT}

The study was approved by the Institutional Ethics Committee of the Fourth Military Medical University (Date: Oct 2016; institutional review board number: TDLL-2016196-450). In December 2017, a 9-year-old girl with bronchomalacia was admitted to our department with a history of pulmonary wheezing and repeated lung infections. The patient received 3 bronchoscopic treatments successively using an endotracheal silicone stent 3 years previously. However, all 3 stents were excreted when the patient had severe cough within 1 month after the intervention.

After admission, computed tomography of the chest with slice thickness at $1 \mathrm{~mm}$ showed that the angular deformities between the left pulmonary artery and the thoracic aorta our previous study.

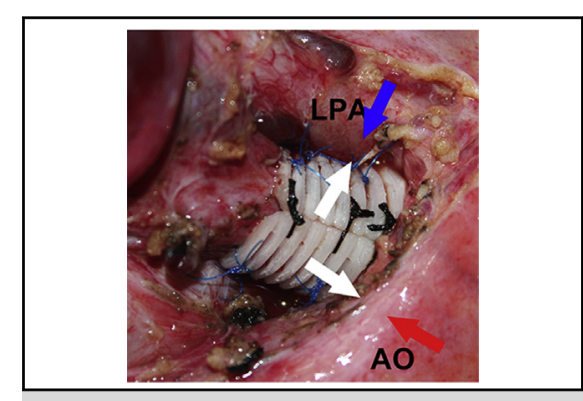

The 3DP PCL scaffold was placed and fixed around the malacic bronchus.

Central Message

A pediatric patient with bronchomalacia received a bronchus suspension operation using 3-dimensional printed polycaprolactone scaffold.

See Commentary on page e291.

pressed against the left mainstem bronchus (Figure 1, $A$ and $B$ ). Further bronchoscope observation showed severe malacic stenosis in the left mainstem bronchus (Figure 1, C). Considering the unsuccessful treatment using the endotracheal stent and the patient's body growth, we developed a bronchus suspension using an individualized 3DP PCL scaffold (Figure 1, D and $E$ ) to resist pressure from both the pulmonary artery and the thoracic aorta. The manufacturing process of PCL scaffold is reported in

The patient was intubated in the supine position and reversed in the right lateral horizontal position. A posterolateral thoracotomy in the fourth intercostal space was performed. Angular deformities in the 2 main arteries were confirmed (Figure 1, F). The 3DP PCL scaffold was placed around the outside of the malacic bronchus. Using 4-0 Polyglactin (Ethicon, Somerville, NJ) sutures, we repaired and suspended the malacic bronchus on the inner surface of the scaffold (Figure 1, $G$ and $H$ ). The patient was extubated in the operating room, transferred to intensive care unit, and discharged a week later.

Repeated computed tomography of the chest and magnetic resonance imaging showed that the space between the left pulmonary artery and the aorta was significantly expanded by the scaffold, although the left mainstem bronchus was still showing malacic stenosis in the field of 

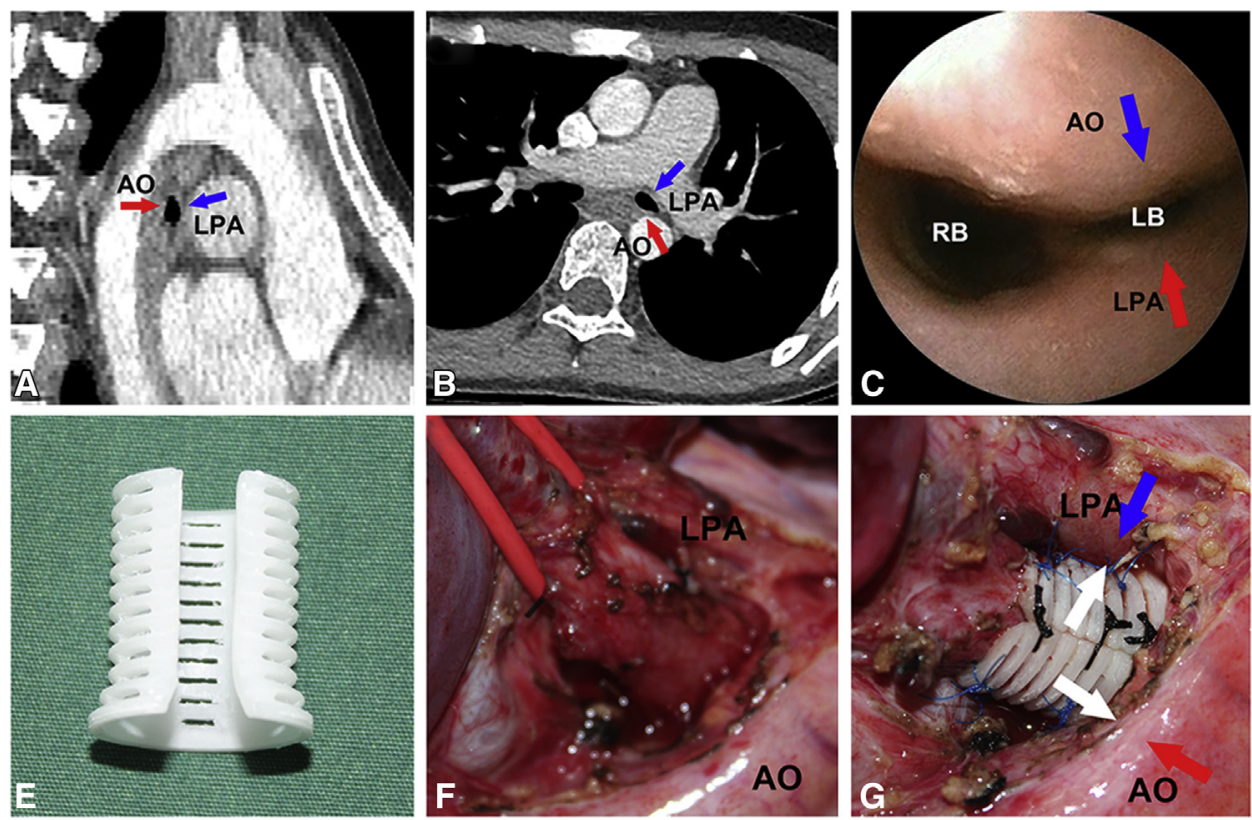
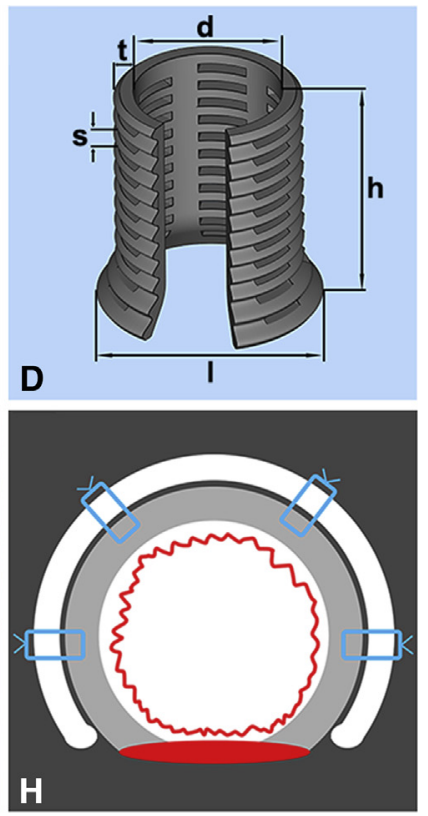

FIGURE 1. The sagittal (A) and horizontal (B) images of chest computed tomography showed that the angular deformities between the LPA and the AO pressed against the left mainstem bronchus. Flexible bronchoscope indicated severe malacic stenosis of left mainstem bronchus (C). The schematic diagram (D) presented dimensions of 3DP PCL (C6H10O2)n scaffold ( $\mathrm{d}=11 \mathrm{~mm}, \mathrm{~h}=18 \mathrm{~mm}, \mathrm{l}=16 \mathrm{~mm}, \mathrm{t}=1 \mathrm{~mm}, \mathrm{~s}=2 \mathrm{~mm}$ ). Considering the small difference of bilateral mainstem bronchus, the internal diameter of the scaffold was designed $0.2 \mathrm{~cm}$ larger than the external diameter of contralateral bronchus. 3DP technique of fused deposition modeling was applied to fabricate the PCL scaffold (E). The raw PCL material was purchased from Daigang Biomaterial Co, Ltd (Jinan, China). The molecular weight was $80,000 \mathrm{Da}$, and the intrinsic viscosity number was 0.5 to $1.0 \mathrm{dL} / \mathrm{g}$; The visual image (F) was taken during the process of operation when the malacic bronchus, LPA, and the AO were dissected away from surrounding organs. The visual image (G) was taken during the same process when the 3DP PCL scaffold was placed and fixed around the malacic bronchus. The illustration (H) showed the method to suspend sutures between the wall and the scaffold. The walls of the bronchus were suspended to the scaffold via sutures placed through designed suture hole interstices at the 2-, 5-, 8-, and 10-o'clock positions. Two rows of sutures with a pitch of $0.5 \mathrm{~cm}$ were fixed in all. In the whole figure, the red and blue arrows, respectively, denote pressure from AO and LPA. The white arrows designate the resistance of scaffold. LB and RB represent left mainstem bronchus and right mainstem bronchus, respectively. $L P A$, Left pulmonary artery; $A O$, thoracic aorta; $R B$, right mainstem bronchus; $L B$, left mainstem bronchus.

bronchoscope (Figure 2, $A-C$ ). One month after surgery, the left mainstem bronchus became broader than before (Figure 2, D-F). Nine months after surgery, the density of scaffold was reduced and the scaffold structure was slowly disintegrating (Figure 2, G-I).

\section{DISCUSSION}

Congenital bronchomalacia is usually associated with other thoracic lesions such as vascular malformation or tumors that may cause tracheal compression. ${ }^{4}$ Relieving the compression in time is the key procedure in the treatment. Traditional surgical options for relieving compression such as aortopexy are often high risk and pose complications. In this case, the bronchoscopic treatment using an endotracheal stent was unsuccessful because the stent cannot support the vascular compression firmly. Also, the stent can easily migrate due to the smooth inner surface of the bronchus. As with cases reported previously, ${ }^{2} 3 \mathrm{DP}$ technology provided an individualized scaffold that could be firmly suspended around the bronchus to support vascular compression. However, the bronchoscopy showed worsening obstruction of the left bronchus 1 week after surgery. This may be caused by the edema of bronchial mucous membrane after surgery. The excision and suture in surgery may lead to edema of bronchial mucous in perioperative period. In the following period, it can be observed that the malacic bronchus gradually expanded due to the self-healing capacity once the compression was eased (Video 1). In theory, PCL with a molecular weight above 65,000 Da can stably exist 2 years in vivo, then gradually degrade into $\mathrm{H}_{2} \mathrm{O}$ and $\mathrm{CO}_{2}$, which can create a local fibrosis around the bronchus. ${ }^{3}$ The hard, fibrous tissues will support the vascular compression in the future. The degradation of PCL scaffold can adapt to 

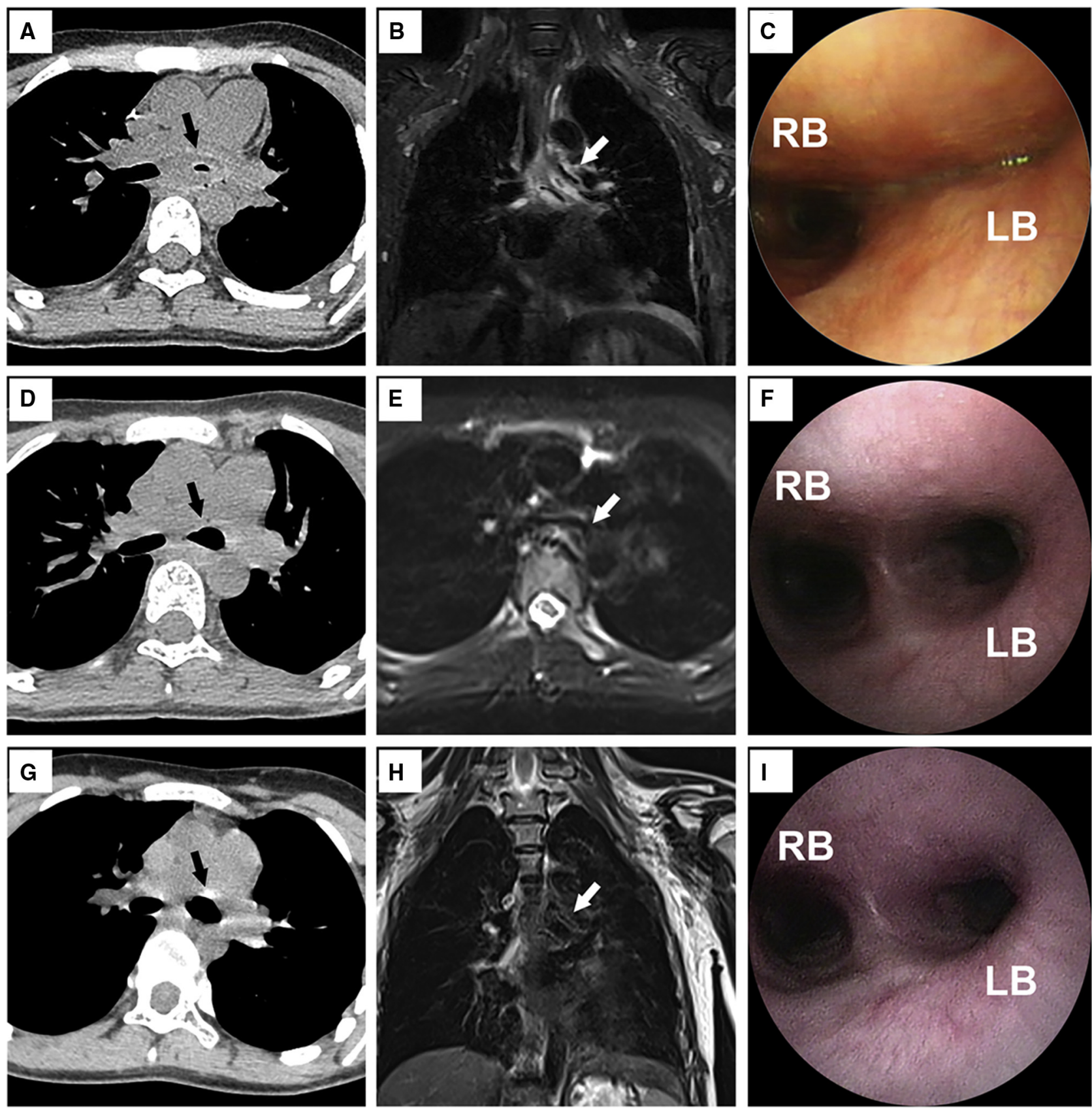

FIGURE 2. CT of the head (A), MRI (B), and bronchoscopic images (C) of the patient showed worsening obstruction of left bronchus 1 week after surgery; CT of the chest (D), MRI (E), and bronchoscopic images (F) presented that the left mainstem bronchus became significantly broadened 1 month after surgery; CT of the chest (G), MRI (H), and bronchoscopic images (I) of the patient showed the broadening of left mainstem bronchus 9 months after surgery. It is worth noting that the density of scaffold was gradually reduced (black arrow in A, D, G) and the structure of scaffold slowly disintegrated (white arrow in B, E, H). LB and RB represent left mainstem bronchus and right mainstem bronchus, respectively.

the growth of the patient. It is, therefore, appropriate for infants. Regarding adult-phenotype bronchomalacia, which is always persistent and progressive, the permanent scaffold may be a better choice. ${ }^{5}$ For cicatricial stenosis caused by tuberculosis or trauma, this method may not be suitable. Until now, the 3DP PCL scaffold has only 


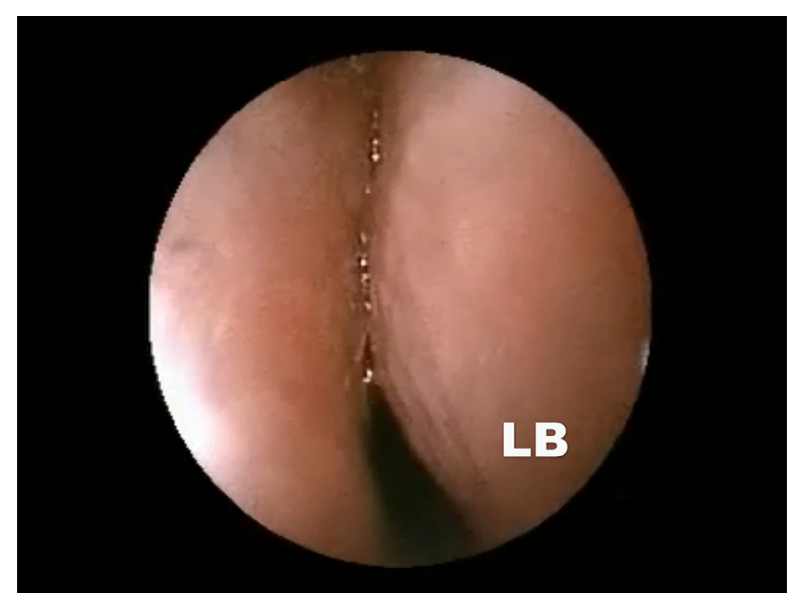

VIDEO 1. The bronchoscopic video shows the malacic trachea of the patient at different periods of the treatment. Video available at: https://www. jtcvs.org/article/S0022-5223(18)33241-0/fulltext. been used to treat tracheomalacia ${ }^{3}$ and bronchomalacia. $^{2}$ It is unknown whether the method can be applied to other locations. In conclusion, 3DP PCL scaffold suspension is an effective treatment for bronchomalacia.

\section{References}

1. Zopf DA, Hollister SJ, Nelson ME, Ohye RG, Green GE. Bioresorbable airway splint created with a three-dimensional printer. N Engl J Med. 2013; 368:2043-5.

2. Morrison RJ, Hollister SJ, Niedner MF, Mahani MG, Park AH, Mehta DK, et al. Mitigation of tracheobronchomalacia with 3D-printed personalized medical devices in pediatric patients. Sci Transl Med. 2015;7:285ra64.

3. Huang L, Wang L, He J, Zhao J, Zhong D, Yang G, et al. Tracheal suspension by using 3-dimensional printed personalized scaffold in a patient with tracheomalacia. J Thorac Dis. 2016;8:3323-8.

4. Carden KA, Boiselle PM, Waltz DA, Ernst A. Tracheomalacia and tracheobronchomalacia in children and adults: an in-depth review. Chest. 2005;127: 984-1005.

5. Morrison RJ, Sengupta S, Flanangan CL, Ohye RG, Hollister SJ, Green GE. Treatment of severe acquired tracheomalacia with a patient-specific, 3D-printed, permanent tracheal splint. JAMA Otolaryngol Head Neck Surg. 2017;143:523-5. 